\title{
Evaluation of Self-Reflection Capacity as a Predictive Factor for Self-stigma levels among Resident Doctors in a Tertiary Care Centre in India
}

\author{
Krishna Patel - Assistant Professor, Department of Psychiatry, CUSMC, India
}

\section{BACKGROUND:}

Resident Doctors are a vulnerable group of population who are responsible for physical as well as mental health of their patients. To act neutrally towards the decision of seeking psychological help for their patients, they themselves ought to be free from self-stigma of seeking psychological help. Finding a factor that would predict the level of self-stigma would be beneficial as we can alter it, to lower the self-stigma levels.

\section{AlMS:}

To evaluate relationship and predictive probability of self stigma level for seeking psychological help with self-reflection capacity and insight amongst resident doctors in a tertiary care center in India.

\section{MATERIALS AND METHODS:}

After taking written informed consent and Institute Ethical Committee permission study was conducted in a tertiary care center in India. Socio-demographic data sheet, Self-Stigma of Seeking Psychological Help Scale, Self-Reflection and Insight Scale were used to collect data, which was followed by analysis using SPSS version 16.

\section{RESULTS:}

Spearman Correlation value of -0.395 between Self-stigma and Self reflection capacity is significant at $p<0.001$. Only $14.7 \%$ variation in self-stigma levels is explained by self-reflection capacity of an individual. Amongst Resident Doctors Odds of having medium level of self stigma is 5.5 times higher in those who have below average Self-reflection capacity as compared to those who have above average Self-reflection capacity.

\section{DISCUSSION:}

Negative and biased attitude towards patients suffering from mental illness is referred as Stigma. ${ }^{[1]}$ And Internalized stigma begins before public stigma affecting the help seeking behavior of an individual.[2] Our results on level of Self-stigma is consistent with the current trend of decrement in stigma levels amongst health professionals. [3] Till date there are no studies that compare self-stigma with the selfreflection capacity. This particular study marks the beginning of search of association between them especially in resident doctors who are in their formative years and hold larger responsibility of directing their patients suffering from mental illness towards appropriate department throughout their career. Lower stigma levels show more readiness to seek psychological help. ${ }^{[4]}$ And hence, resident doctors with lower stigma level can convince their patients to seek help.

\section{CONCLUSION:}

Measures that help in increasing the self reflection capacity and develop insight in an individual can lower the levels of self stigma associated with seeking psychological help. When implemented on resident doctors, it can eventually have a larger impact on future referrals in psychiatry department in health sector.

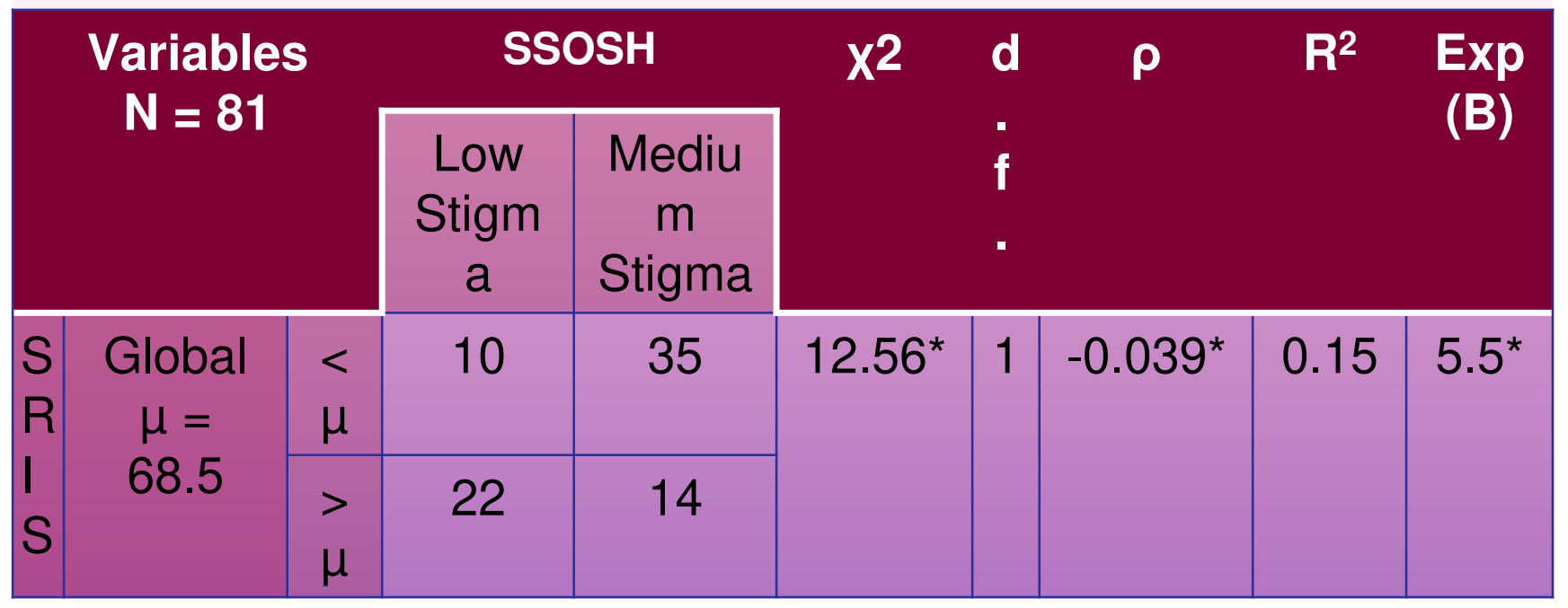

*-p-value $</=0.001$; SSOSH = Self-Stigma Of Seeking Help; SRIS $=$ Self-Reflection and Insight Scale; $\rho=$ Spearman Correlation; $R^{2}$ $=$ Cox \& Snell R Square
Stigma Level Distribution in \%

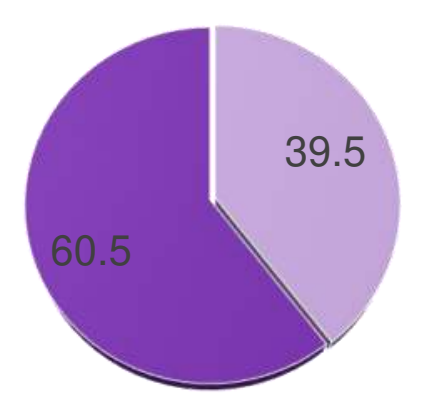

- Low Stigma

- High Stigma
Correlation of Self-stigma with Self-reflection

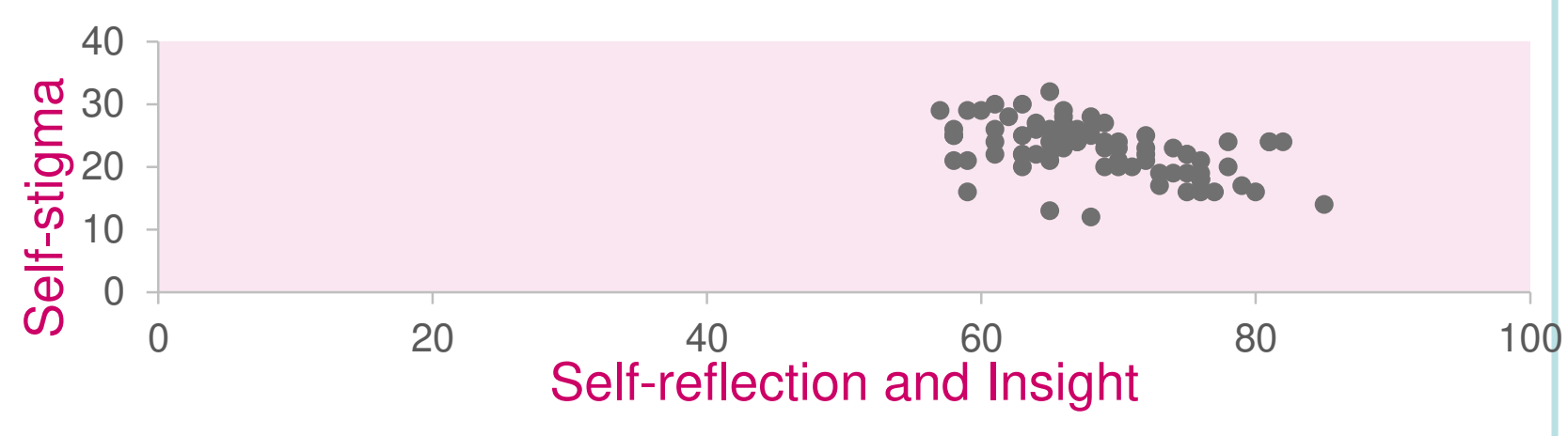

\section{References :}

1.Dovidio, J. F.,Major, B. \& Crocker, J. 2000 Stigma: introduction and overview. InThe Social Psychology of Stigma. pp.1^13.

2.Barney, LJ et al (2006). Stigma about depression and its impact on help-seeking intentions. ANJP, 40, 51-54.

3.Chandramouleeswaran S, et al. Stigma and attitudes towards patient with psychiatric illness among postgraduate Indian physician.

Indian J Psychol Medicine.2017;39(6):748-749

4.Vogel, D. L., Wade, N. G., \& Haake, S. (2006). Measuring the self-stigma associated with seeking psychological help. Journal of

Counseling Psychology, 53, 325-337. doi:10.1037/0022- 0167.53.3.325 